УДК 624.04(075.8)

\title{
РЕШЕНИЕ ЗАДАЧИ ЭЙЛЕРА ОБ УСТОЙЧИВОСТИ СТЕРЖНЯ ЧИСЛЕННО-ГРАФИЧЕСКИМ МЕТОДОМ
}

\author{
Аккулова Мадина Муслимовна \\ Нагоев Айдемир Муратович \\ студенты \\ Научный руководитель: Культербаев Хусен Пшимурзович \\ д.т.н., профессор \\ СКФУ «Северо-Кавказский центр \\ математических исследований»
}

\begin{abstract}
Аннотация: Рассмотрена задача Эйлера об устойчивости сжатого стержня. Применяется метод конечных разностей в сочетании с графическими возможностями программного комплекса Matlab. Рассмотрены тестовый и реальный примеры определения критических сил. Тестовый пример показал точное совпадение критической силы с литературными данными. Результаты подтверждают эффективность и универсальность предлагаемого метода.

Ключевые слова: Задача Эйлера; критическая сила; численнографический метод; математическое моделирование; обыкновенное дифференциальное уравнение; краевые условия.
\end{abstract}

\section{SOLUTION OF EULER'S PROBLEM OF ROD STABILITY BY THE NUMERICAL-GRAPHICAL METHOD}

\section{Akkulova Madina Muslimovna \\ Nagoev Aydemir Muratovich Kulterbaev Khusen Pshimurzovich}

\begin{abstract}
The Euler problem on the stability of a compressed rod is considered. The finite difference method is used in combination with the graphic capabilities of the Matlab software package. Test and real examples of determining critical forces are considered. The test example showed an exact coincidence of the critical force with the literature data. The results confirm the effectiveness and versatility of the proposed method.
\end{abstract}


Key words: Euler's problem; critical power; numerical-graphical method; math modeling; ordinary differential equation; boundary conditions; finite difference method.

\section{Введение}

Сжатые стержни, как элементы строительных сооружений в виде колонн, элементы ферм, рам, а также в конструкциях машиностроения и приборостроения встречаются очень часто. Расчёт их устойчивости является одной из наиболее сложных задач. Поэтому эта тема включена в программы вузов при изучения таких дисциплин как сопротивление материалов, строительная механика, устойчивость и динамика сооружений, техническая механика $[10,11]$.

Устойчивость прямолинейного сжатия стержня впервые была рассмотрена Л. Эйлером в 1744 г., в Петербургской императорской академии, с которой он поддерживал тесные связи в 1766 - 1783 г.г. Сначала результаты Эйлера по определению критических сил не стали популярными для практического применения. В большинстве своём «стержни» были каменными с тремя размерами, сравнимыми между собой и им не угрожала опасность потери устойчивости. Через многие годы, когда появились сначала чугунные, а затем и металлические колонны, идеи Эйлера об устойчивости стержней стали весьма актуальными.

\section{1. Математическое моделирование задачи}

Задача Эйлера об устойчивости прямолинейного сжатого стержня, вскоре стала классической и не потеряла своей актуальности до настоящего времени $[1,3,4,5]$. Она была математически сформулирована как задача на собственные значения для дифференциального уравнения второго порядка

$$
\mathrm{v}^{\prime \prime}(\mathrm{x})+\mathrm{k}^{2} \mathrm{v}(\mathrm{x})=0, \quad \mathrm{v}(0)=\mathrm{v}(l)=0, \quad \mathrm{x} \in[0, l], \quad \mathrm{k}^{2}=\mathrm{F} / \mathrm{b}, \quad \mathrm{b}=\mathrm{EJ} .
$$

Здесь $\mathrm{v}(\mathrm{x})$ - функция изогнутой оси стержня, Е, J-физико-механические параметры системы, F-сжимающая сила. Необходимо отыскать такие её значения (критические силы), при которых возможны ненулевые решения уравнения (1). Большое разнообразие опор требовало повышения порядка дифференциального уравнения (1) до четвертого. Для каждого случая аналитическое решение становится затруднительным, так как на конечном этапе приходится решать систему трансцендентных уравнений для определения собственного значения $\mathrm{k}^{2}$, а значит, и критической силы $\mathrm{F}_{\mathrm{\kappa}}$. Между тем 
имеется возможность решения данной проблемы более универсальным и простым способом с помощью численных методов и компьютерных технологий $[2,6,7,8,9]$.

Уравнение (1) продифференцируем дважды и запишем в виде

$$
\mathrm{v}^{\prime \prime \prime \prime}(\mathrm{x})+\mathrm{k}^{2} \mathrm{v}^{\prime \prime}(\mathrm{x})=0, \quad \mathrm{x} \in(0, l) \text {. }
$$

Основное уравнение (2) дополняется краевыми условиями. На нижнем и верхнем концах условия являются кинематическими: прогибы и углы поворота сечений равны нулю

$$
\mathrm{v}(0)=0, \quad \mathrm{v}^{\prime}(0)=0, \quad \mathrm{v}(l)=0, \quad \mathrm{v}^{\prime}(l)=0 .
$$

Рассмотрим задачу по расчётной схеме рис. 1 , имея целью тестирование предлагаемого численно-графического метода определения критической силы.

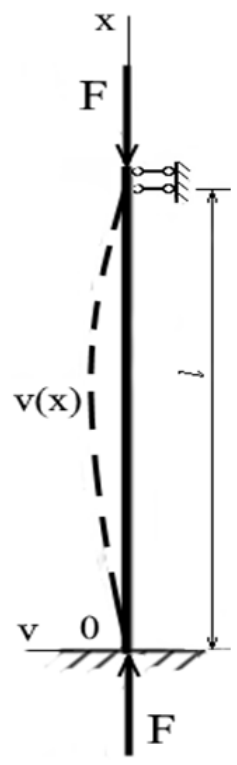

Рис. 1.

Система уравнений (2) - (3) образует математическую модель задачи. Критическая сила в этом случае определяется формулой, найденной из системы трансцендентных уравнений $[1,3]$

$$
\mathrm{F}_{\mathrm{K}}=\frac{4 \pi^{2} \mathrm{~b}}{l^{2}} .
$$

\section{2. Алгоритм численно-графического метода}

Перейдем теперь от аналитической математической модели к численной с помощью метода конечных разностей. С этой целью заменим в (2) область непрерывного изменения аргумента областью дискретного изменения с шагом $\mathrm{h}=l /(\mathrm{n}-1)$ 


$$
\mathrm{L}_{\mathrm{h}}=\left\{\mathrm{x}_{\mathrm{i}}=(\mathrm{i}-1) \mathrm{h}, \quad \mathrm{i}=1,2, \ldots, \mathrm{n}\right\} .
$$

Вместо функции $\mathrm{v}\left(\mathrm{x}_{\mathrm{i}}\right)$ будем рассматривать сеточную функцию $\mathrm{y}\left(\mathrm{x}_{\mathrm{i}}\right) \approx \mathrm{v}\left(\mathrm{x}_{\mathrm{i}}\right)$. От дифференциальных операторов в (2) - (5) перейдем к разностным

$$
\begin{aligned}
& \mathrm{v}^{\prime \prime \prime}\left(\mathrm{x}_{\mathrm{i}}\right)=\frac{1}{\mathrm{~h}^{4}}\left(\mathrm{y}_{\mathrm{i}-2}-4 \mathrm{y}_{\mathrm{i}-1}+6 \mathrm{y}_{\mathrm{i}}-4 \mathrm{y}_{\mathrm{i}+1}+\mathrm{y}_{\mathrm{i}+2}\right), \quad \mathrm{i}=3,4, \ldots \ldots, \mathrm{n}-2, \\
& \mathrm{v}(0)=y_{1}, \quad \mathrm{v}^{\prime \prime}\left(\mathrm{x}_{\mathrm{i}}\right) \approx \frac{1}{\mathrm{~h}^{2}}\left(\mathrm{y}_{\mathrm{i}-1}-2 \mathrm{y}_{\mathrm{i}}+\mathrm{y}_{\mathrm{i}+1}\right), \quad \mathrm{v}(l)=\mathrm{y}_{1} . \\
& \mathrm{v}^{\prime}(0) \approx \frac{1}{2 \mathrm{~h}}\left(-3 \mathrm{y}_{1}+4 \mathrm{y}_{2}-\mathrm{y}_{3}\right), \quad \mathrm{v}^{\prime}(l) \approx \frac{1}{2 \mathrm{~h}}\left(\mathrm{y}_{\mathrm{n}-2}-4 \mathrm{y}_{\mathrm{n}-1}+3 \mathrm{y}_{\mathrm{n}}\right),
\end{aligned}
$$

С помощью представлений (5) и несложных преобразований основное уравнение (2) для внутрисеточных точек примет вид

$$
\begin{aligned}
& \mathrm{y}_{\mathrm{i}-2}+\mathrm{a} \mathrm{y}_{\mathrm{i}-1}+\mathrm{by}_{\mathrm{i}}+\mathrm{a} \mathrm{y}_{\mathrm{i}+1}+\mathrm{y}_{\mathrm{i}+2}=0, \quad \mathrm{i}=3,4, \ldots, \mathrm{n}-2 \text {. } \\
& \mathrm{a}=\mathrm{k}^{2} \mathrm{~h}^{2}-4, \quad \mathrm{~g}=6-2 \mathrm{k}^{2} \mathrm{~h}^{2} .
\end{aligned}
$$

Аналогично вместо граничных условий (3) получим

$$
\mathrm{y}_{1}=0,-3 \mathrm{y}_{1}+4 \mathrm{y}_{2}-\mathrm{y}_{3}=0, \quad \mathrm{y}_{\mathrm{n}-2}-4 \mathrm{y}_{\mathrm{n}-1}+3 \mathrm{y}_{\mathrm{n}}=0, \quad \mathrm{y}_{\mathrm{n}}=0 \text {. }
$$

Рассмотрим тестовый численный пример. Возьмем для тестирования предлагаемого алгоритма стержень по рис. 1.

Пример 1. Пусть $\mathrm{b}=4, \quad l=\pi$.

В таком случае критическая сила определяется формулой (4). Обратим внимание, что параметры заданы в безразмерных величинах. Поэтому получаемые результаты также будут отвлечёнными числами. Уравнения (6), (7) перепишем в матрично - векторной форме

$$
\mathrm{CY}=0 \text {. }
$$

Здесь С - квадратная матрица порядка $\mathrm{n}, \mathrm{Y}$ - вектор-столбец. В развёрнутом виде (8) принимает вид

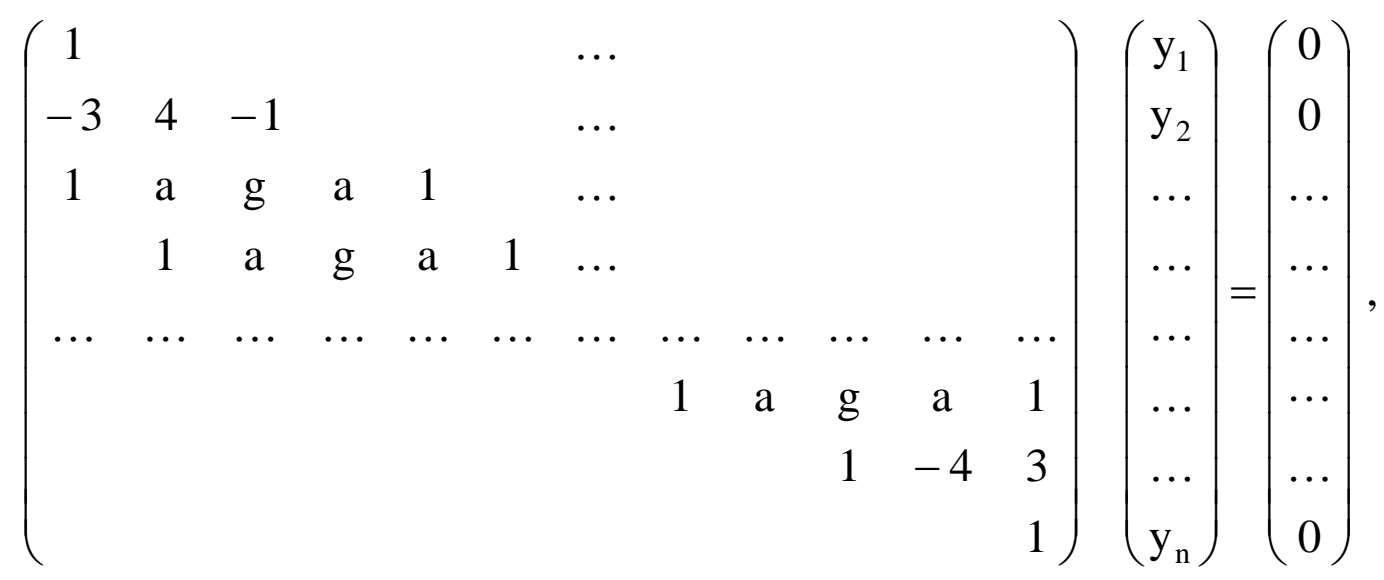


$\mathrm{Y}=\left\{\mathrm{y}_{1}, \mathrm{y}_{2}, \ldots, \mathrm{y}_{\mathrm{n}}\right\}_{\mathrm{T}}$.

Знак Т в верхнем индексе соответствует транспонированию вектора. Нулевые элементы матрицы и вектора не выписаны.

Система уравнений (8) имеет тривиальное решение

$\mathrm{Y}=0$,

которое не представляет практического интереса, так как соответствует исходной прямолинейной форме равновесия стержня. Задача состоит в том, чтобы найти собственные значения $\mathrm{F}$ матрицы $\mathrm{C}$, при которых возможны ненулевые решения. Это требование приводит к характеристическому уравнению

$\operatorname{det} \mathrm{C}(\mathrm{F})=0$.

При больших значениях $\mathrm{n}$ развертывание определителя при неизвестном значении F, а далее решение уравнения (10) представляет большие сложности.

Возникающие проблемы легко преодолеваются, если воспользоваться возможностями современной вычислительной техники и прикладных программных комплексов (Matlab, MathCad и т.д.). Они позволяют легко визуализировать функцию в левой части (10) на экране монитора в координатной системе $\mathrm{F}-\operatorname{det} \mathrm{C}(\mathrm{F})$. Тогда точки пересечения соответствующей кривой с осью F совпадают с искомыми значениями критической силы.

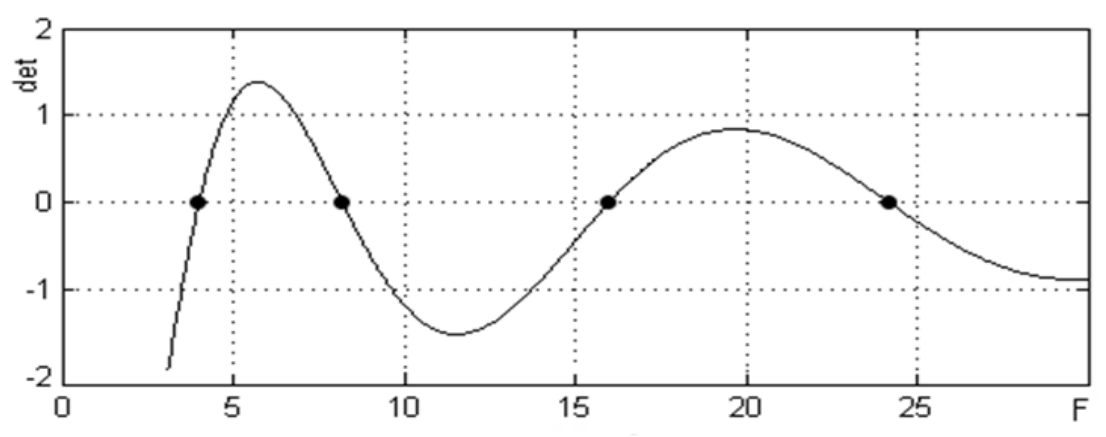

Рис. 2.

Разработана компьютерная программа на языке Matlab и проведены вычисления, результаты которых, прочитанные с экрана монитора (рис. 2), совпадают с точными, а именно, отличаются от них на почти неразличимую величину порядка 0,00001 . Отсюда вывод: численный метод является простым и эффективным средством определения собственных значений для дифференциальных уравнений. 
Точки пересечения графика с осью F дали следующие первые четыре элемента спектра критических сил:

$$
F=\{4,000 ; 8,183 ; 16,000 ; 24,187\} .
$$

Первая критическая сила, наиболее важная в практических приложениях, точно совпадает с результатом по формуле (4).

Теперь рассмотрим реальную строительную колонну из стального двутавра, работающую по расчётной схеме рис. 1 .

Пример 2. Дано: двутавр №16, $\mathrm{J}_{\min }=58,6, \mathrm{~cm}^{4}, \mathrm{E}=2,1 \cdot 10^{5} \mathrm{MПа}, l=12 \mathrm{м}$.

По этим данным получен график рис. 3.

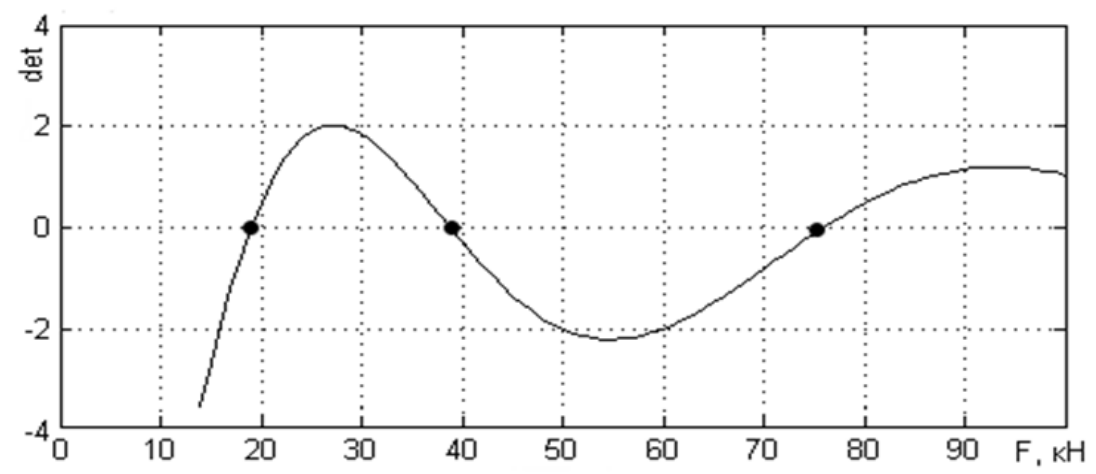

Рис. 3.

\section{Выводы}

1. Численно-графический метод определения критических сил сжатых стержней в сочетании с вычислительным комплексом Matlab является простым и универсальным средством решения задач Эйлера.

2. Численно-аналитический метод не связан с необходимостью составления и решения систем трансцендентных уравнений и обладает свойствами универсальности.

3. Примеры показывают, что метод и соответствующие компьютерные программы могут легко адаптироваться к неклассическим граничным условиям, в то время как аналитические решения требуют значительных усилий для каждой новой задачи. 


\section{Список литературы}

1. Вольмир А.С. Устойчивость деформируемых систем. М.: Наука. $1967.984 \mathrm{c}$.

2. Самарский А.А., Гулин А.В. Численные методы. М.: Наука, Гл. ред. физ.-мат. лит.,1989. - 432 с.

3. Алфутов Н.А. Основы расчёта на устойчивость упругих систем. М.: Машиностроение. 1978. 312c.

4. Прочность, устойчивость, колебания. Справочник в трёх томах. Том 3. Под редакцией Биргера И.А., Пановко Я.Г. М.: Машиностроение. 1968. 567 с.

5. Боднарь Т.А. Численный анализ устойчивости нагруженного стержня переменной жёсткости // Вычислительные технологии. СОН. РАН. 2003. Т. 8. №2. c. 27-35.

6. Варвак П.М., Варвак Л.П. Метод сеток в задачах расчёта строительных конструкций. М.: Стройиздат, 1977. 154 с.

7. Вержбицкий В.М. Основы численных методов. М.:Высшая школа, $2002.840 \mathrm{c}$.

8. Ильин В.П., Карпов В.В., Масленников А.М. Численные методы решения задач строительной механики.-М.: Изд-во ACB; СПб.:СПбГАСУ,2005.-425 с.

9. Караманский Т.Д. Численные методы строительной механики. -М.: Стройиздат, 1981. -436 с.

10. Масленников А.М. Динамика и устойчивость сооружений. Учебник и практикум для вузов. -М. : Издательство Юрайт. 2016. -366 с.

11. Феодосьев В.И. Сопротивление материалов. -М.: Изд. МГТУ им. Н.Э. Баумана, 2003. -592 с.

12. Kulterbaev Kh.P., Baragunova L.A., Shogenova M.M., Senov Kh. M. About a High-Precision Graphoanalytical Method of Determination of Critical Forces of an Oblate Rod. Proceedings 2018 IEEE International Conference "Quality Management, Transport and Information Security, Information Technologies" (IT\&QM\&IS). September,24-28,2018. St. Petersburg . Russia 2018. P. 794-796 c. 